\title{
Periferias en Cali: alfabetización digital con grupos sociales subalternos en medios sociales digitales
}

\section{Peripheries in Cali: Digital Literacy with Subaltern Social Groups in Digital Social Media}

\author{
Eliana Noscué Mera \\ Universidad Nacional de La Plata \\ Facultad de Periodismo y Comunicación Social \\ Sede 44 e/ 8 y 9 \#676 - Oficina 12 \\ La Plata, Buenos Aires \\ eliananoskwe@gmail.com \\ Argentina
}

\begin{abstract}
Resumen:
El presente artículo describe las experiencias relacionadas con la construcción de representaciones socioculturales y mediáticas en los medios sociales digitales (Facebook, Instagram, YouTube) durante el desarrollo del proceso pedagógico de alfabetización digital denominado Alto Nápoles en Red. Esta técnica metodológica cualitativa se desarrolló con una comunidad migrante involuntaria y en situación de desplazamiento forzado proveniente de las zonas rurales del suroccidente colombiano (Departamento del Cauca) y radicada en una de las periferias de Santiago de Cali. Se trata de dar cuenta sobre las posibilidades, las resistencias y los obstáculos históricos que han transitado estos grupos sociales subalternos en el marco del conflicto interno armado en Colombia. El objetivo es conocer sobre los usos y apropiaciones de las Tecnologías de Información y Comunicación (TIC) digitales a partir de las diferencias culturales, las desigualdades socioeconómicas y las desconexiones mediáticas.
\end{abstract}

Palabras clave: Grupos sociales subalternos; alfabetización digital; representaciones; periferias; medios sociales digitales.

\begin{abstract}
:
This article describes experiences related to the construction of sociocultural and media representations in digital social media (Facebook, Instagram, YouTube) during the development of a digital literacy pedagogical process called Alto Nápoles en Red. This qualitative methodological technique was developed with an involuntary migrant community in a situation of forced displacement from the rural areas of southwestern Colombia (Department of Cauca) and located in one of the peripheries of Santiago de Cali. The article provides an account of the possibilities, resistances and historical obstacles that these subaltern social groups have experienced within the framework of the internal armed conflict in Colombia. The aim is to know about the uses and appropriations of digital Information and Communication Technologies (ICT) based on cultural differences, socioeconomic inequalities and media disconnections.
\end{abstract}

Keywords: Subaltern social groups; digital literacy; representations; peripheries; digital social media.

Recibido: 08/04/2019 | Aceptado: 10/06/2019 | Publicado: 18/06/2019

| pág. 70 - 95 
Periferias en Cali: alfabetización digital en grupos sociales subalternos en medios sociales digitales

\section{Introducción}

El suroccidente, región a la cual pertenece Santiago de Cali, ha sido una de las zonas de Colombia más afectadas por el conflicto interno armado que se llevó a cabo a lo largo de la segunda mitad del siglo XX; el cual generó desde 1985 hasta la actualidad 7 millones 700 mil desplazados forzados internos a nivel nacional pertenecientes a comunidades indígenas, afrodescendientes y campesinas mestizas ("Colombia sigue siendo el país con más desplazados internos...”, 2017). Los departamentos del Cauca, Nariño, Choco y Valle del Cauca que componen esta zona se consolidaron como expulsores del $40 \%$ de poblaciones desplazadas ("Los departamentos de Colombia con mayor número de desplazados”, 2016). De este porcentaje una gran mayoría llega a Cali convirtiéndola en una de las receptoras más representativas de este flagelo. En el año 2012 se indicó que, a la fecha, más de 80 mil personas registradas oficialmente como desplazadas residían en esta ciudad ("De cuatro millones de desplazados en Colombia...”, 2012). ${ }^{1}$

Así, este territorio se ha configurado en una ciudad multicultural desde la desigualdad, la diferencia y la desconexión (García Canclini, 2004). Estos grupos sociales subalternos ${ }^{2}$ como se reconocen aquí por haber sido parte de diversas luchas sociopolíticas e históricas que los direccionaron a su situación actual; se han asentado en periferias reconocidas por altos niveles de pobreza y connotaciones negativas construidas en torno a las zonas territoriales y a las poblaciones que las habitan.

Debido a la amplia diversidad cultural y a la necesidad de reconocimiento de derechos que se le debe históricamente a estas comunidades, ha sido indispensable 
preguntar sobre el estado actual de las representaciones socioculturales ${ }^{3}$ que pudiesen estar construyendo en los medios sociales. Es decir, la construcción de memoria histórica en constante deconstrucción y reconstrucción identitaria; a partir de los usos y apropiaciones de las TIC digitales que aporte a la representación mediática ${ }^{4}$ de la ciudad donde se incluya las periferias y sus formas de vida. Aquellas que generalmente son excluidas de los folletos turísticos y del discurso institucional hegemónico.

Esta pregunta se plantea en el marco de una investigación doctoral en comunicación que propone un estudio exploratorio analítico sobre los usos y apropiaciones de las TIC digitales, vinculadas a la producción, difusión y visualización de productos audiovisuales en los medios sociales, por parte de estas poblaciones.

A partir de la metodología aplicada fue posible conocer sobre las características de acceso a los dispositivos y a las conexiones que les permite, intermitentemente, ser parte de la sociedad mediatizada aunque desde las diferencias históricas que los definen. En ese sentido fue importante encontrar, que si bien el rol de prosumidores ${ }^{5}$ es incipiente por las desigualdades de acceso y/o alfabetización digital; ${ }^{6}$ las acciones que llevan a cabo en estos espacios y con estas herramientas poseen amplias referencias a sus recursos simbólicos y biográficos; es decir, a sus historias de vida antes del desplazamiento y/o la migración, a su cosmovisión, saberes ancestrales relacionados con la gastronomía, la organización comunitaria, con sus formas de vida rurales que ahora son parte de las periferias urbanas. La experiencia pedagógica desarrollada evidenció que, al atender la importancia de inclusión digital por parte de Estados y gobiernos, existen altísimas posibilidades; seguro que no para acabar con la amplísima brecha digital que está relacionada con las desigualdades históricas de las que han sido parte estas comunidades, pero sí para garantizar mínimamente el derecho a la información y la comunicación que actualmente es transversal a los medios sociales digitales. 
Periferias en Cali: alfabetización digital en grupos sociales subalternos en medios sociales digitales

Noscué Mera

|Discusión teórico-empírica: redes virtuales $\neq$ redes comunitarias

Alto Nápoles es una amplia zona periférica ubicada al suroccidente de la ciudad y compuesta por distintos barrios que se fueron conformando irregularmente durante el último cuarto del siglo $\mathrm{XX}$ y lo que va del XXI. Esta conformación social y territorial ha sido caracterizada por aspectos rurales que traen consigo los grupos subalternos que migran involuntariamente $\mathrm{y} / \mathrm{o}$ se desplazan forzosamente desde el suroccidente colombiano, principalmente desde el departamento del Cauca.

La comunidad puntual con la que se desarrolla esta investigación proviene de municipios del Departamento del Cauca tales como Buenos Aires, Caldono y La Vega. En estas zonas existen diversos cabildos indígenas por lo que muchas de las personas con las que se trabajó pertenecen a las comunidades indígenas Yanacona y Nasa. El sector donde se asentaron en la zona periférica caleña es denominado por ellos mismos como Brisas de Alto Nápoles; así mismo es necesario mencionar la zona denominada como
Bosques 1 y 2, ya que es la zona aledaña y en conjunto suman 2 mil personas aproximadamente. Cabe aclarar Bosques 1 y 2 cuenta con 20 años de historia y Brisas de Alto Nápoles con una más reciente de 8 años.

Esta última experiencia de conformación territorial, por estar más cerca en el tiempo, permitió conocer por relatos de sus protagonistas los diversos procesos que han llevado a cabo para la construcción de sus casas, la incorporación de la energía, el alcantarillado, el agua y la pavimentación de calles y andenes. Para lograrlo, esta comunidad prioriza la minga o minka ${ }^{7}$ que implica la cooperación de todos los habitantes en una actividad de trabajo que conlleve a un bien común.

Gracias a las mingas, en 8 años, Brisas de Alto Nápoles pasó de ser una ladera inhabitada a una zona en constante construcción territorial e identitaria que emana la ruralidad y las prácticas culturales propias de los pueblos campesinos; aunque estén ubicados a 15 minutos de una de las principales autopistas de la ciudad. 
Por todas estas características es necesario problematizar la generación de conocimiento sobre los usos y apropiaciones de las TIC, ya que "gran parte de la producción académica que las vincula con el territorio tiene como ámbito de observación y análisis a las áreas metropolitanas de economías avanzadas" (Vio y Fritzsche, 2007, p. 64) y no se vincula las experiencias que se viven $y$ se proponen desde las periferias. De todas formas, no se trata de debatir sobre "el lugar central de la producción y también del intercambio de información" (Vio y Fritzsche, 2007, p. 50) con el que es percibida la ciudad y su centro de producción al utilizar las TIC como herramientas para la acumulación de capital.

Se trata más bien de conocer, en primera instancia, las características propias de un territorio en construcción y sin reconocimiento gubernamental que evidencia los flagelos sociales más profundos con los que han convivido los colombianos excluidos: la pobreza económica, la falta de educación formal, la falta de infraestructura y servicios básicos; pero también la evidencia de la organización social y comunitaria que sobrevive al desplazamiento y al cambio abrupto de su territorio natal y que se encuentra inscripto en la memoria histórica de estos pueblos.

En ese sentido es necesario resaltar la importancia que tiene el término de red en este estudio que, según Quintar (2007, p. 72), en las ciencias sociales “... es inicialmente utilizado por los análisis acerca de los mecanismos de supervivencia básicos desarrollados por grupos poblacionales marginados." También, apunta Quintar, esta noción se relaciona con la articulación de movimientos sociales vinculados a los campos políticos y culturales, para consolidarse en redes mucho más amplias que pueden llegar a constituirse en redes internacionales.

Este tipo de organización política y social, propia de la noción de red, es la que se aplica empíricamente en esta comunidad. Cuestiones que aportan al territorio una hibridación cultural (la relación corporal con la música, la estética, las relaciones familiares y sociales) e histórica; sin embargo, las 
Periferias en Cali: alfabetización digital en grupos sociales subalternos en medios sociales digitales

diferencias que existen entre estos grupos sociales (indígenas, afrodescendientes, campesinos mestizos) pasan a un segundo plano, al priorizar las necesidades comunes del territorio. Es característico en la red de trabajo comunitario que se ha conformado que la producción sea lenta en comparación a los modos de producción del sistema capitalista y neoliberal; ya que los trabajos comunitarios se realizan los domingos, día en el que se encuentran descansando de sus trabajos no calificados 0 desconectados de la sociedad y la economía de la información tales como los empleos de vigilantes, obreros rasos, empleadas de servicio doméstico y vendedores ambulantes.

Pues bien, sería factible pensar que estas propiedades teóricas y empíricas sobre la red comunitaria offline son fácilmente extrapolables a la noción de red digital; tal cual como lo señalaba Tim Berners-Lee, el creador de la Red World Wide Web más conocida como la Web 1.0 o la Web pasiva. Esto teniendo en cuenta una entrevista del año 2000 donde resaltaba que el "carácter abierto y descentralizado de la Red permitió que la misma se expandiera rápidamente sin necesidad de contar con alguna autoridad centralizada para desarrollarse” (Quintar, 2007, p. 76).

Así entonces, "la creencia de que la web 2.0 era un espacio comunitario y de colaboración inspiró en aquellos tiempos a muchos entusiastas a trabajar en la construcción de distintas plataformas, y algunos ecos de este espíritu resuenan aún hoy" (Van Dijck, 2016, p. 28). Sin embargo, el nuevo milenio trajo consigo la infraestructura de la Web 2.0 o la Web Social y con ella pocas empresas que posteriormente se convirtieron en corporaciones (Google, Apple, Amazon, Facebook) dedicadas a la producción de plataformas propias que se ofrecieron para ser "las intermediarias para la trasmisión de datos de comunicación y de información” (Van Dijck, 2016, p. 21).

Fue así como se achicó la esperanza democratizadora que los medios sociales traían consigo para superar la temible homogeneización social de los medios masivos y hegemónicos del siglo XX. Tanto se ha achicado el 
enfoque de la producción colaborativa y comunicación participativa que los avances tecnológicos que se tienen de la Web 3.0 o la Web semántica están intrínsecamente relacionados con la monetización de los datos y los metadatos de los usuarios a nivel global, es decir, la utilización de estos, autorizados por el usuario, con fines de explotación comercial.

A pesar de esta capitalización de los medios sociales, o conectivos como prefiere llamarlos (Van Dijck, 2016), se coincide aquí con las y los investigadores que sostienen que "las plataformas de medios sociales han introducido un espacio dentro del cual se desdibujan los límites entre lo público y lo privado" asegurando que "esta imprecisión abre nuevas posibilidades para la conformación de identidades" (Van Dijck, 2016, p. 37) y subsecuentes representaciones sociales $\mathrm{y}$ mediáticas.

\section{|Las diferencias y las desigualdades}

Hace más de una década Néstor García Canclini propuso un análisis que articuló tres nociones claves en las ciencias sociales cuando de procesos culturales se trata. Problematizó las antropologías de las diferencias, las teorías sociológicas de la desigualdad y los estudios comunicacionales que estudian las conexiones y las desconexiones mediáticas.

En ese sentido, fue fundamental comprender que es más importante "prestar atención a las mezclas y los malentendidos que vinculan a los grupos” (García Canclini, 2004, p. 21) que comparar las diferencias $\mathrm{y}$ justificarlas en el relativismo cultural.

Entonces lo de aprender a ser interculturales, en el proceso que se expone aquí, fue una premisa que conllevó a tener muy en cuenta cómo dicha comunidad ha construido su red comunitaria. Todo ello reconociendo que es una comunidad culturalmente híbrida. En apariencia se podría pensar que, al poseer condiciones económicas y sociales similares, era factible la existencia de una armonía política si se considera que su organización comunitaria tiene muy claro sus objetivos tangibles. Sin embargo, las complejidades culturales que 
pudiesen impedir una organización comunitaria más sólida estaban relacionadas con la alteridad. La dificultad de reconocimiento de las particularidades culturales de quienes provienen de las zonas rurales y los cabildos indígenas y que devienen en formas y maneras propias de organización política y ejecución de proyectos.

Como se dijo, la zona Bosques lleva mucho más tiempo de constitución que Brisas de Alto Nápoles. Es por ello que los habitantes de Bosques, campesinos mestizos y ciudadanos periféricos con mucho más tiempo de convivencia en la ciudad, califican como extrañas las formas de organización política de los indígenas llegados hace ocho años. Pues estos últimos, a partir de sus tradiciones político-culturales, proponen organizaciones cabildantes o de comisiones para facilitar las mejoras territoriales. Esto es desconocido u olvidado por quienes han aprehendido formas de acción o inacción política urbana, es decir, consideraciones más individualistas. Por ello, los líderes de Bosques manifestaron la necesidad de que los indígenas abandonaran sus formas de organización y aprehendieran, como ellos lo hicieron, las nuevas dinámicas, que según ellos exige la ciudad.

Es así como se evidencia que al interior de estas dos zonas es posible ubicar cinco subgrupos sociales. Por una parte los vecinos mestizos que son representados por líderes comunitarios históricos que han hecho parte de las Juntas de Acción $\mathrm{Comunal}^{8}$, el Cabildo Nasa de Alto Nápoles $^{9}$ que representa la comunidad Nasa de la zona, los líderes de Brisas de Alto Nápoles que es la zona habitada mayoritariamente por indígenas del pueblo Yanacona ${ }^{10}$, los afrodescendientes que son familias numerosas y los autodenominados organizados, que se refiere a un grupo compuesto por indígenas, campesinos mestizos $y$ afros que viven juntos en una zona determinada.

Estas disimilitudes generadoras de tensiones y desencuentros al interior de esta red comunitaria evidencian lo que García Canclini (2004) denomina una "problemática de la diferencia", visible principalmente en las prácticas culturales y políticas: 
La absolutización se presenta con dos movimientos, por una parte, se distinguen como exclusivos de los pueblos indígenas sus lenguas y ciertos "valores": la reciprocidad de las relaciones comunitarias, el trabajo no remunerado, sistemas normativos propios, relaciones sociales gobernadas por regímenes de autoridad, costumbres alimentarias originadas por el arraigo en el territorio tradicionalmente ocupado por cada grupo. Al mismo tiempo, se define a esos rasgos como inalterables y se actúa para darles continuidad (García Canclini, 2004, p. 47).

La desigualdad social que une a estos subgrupos es fragmentada por las diferencias culturales que los identifica y complejiza el fortalecimiento de ese proceso emergente propio "de proyectos germinales, que son diferentes del proyecto que se está tratando de plantear como homogéneo $y$ como hegemónico" (Bonfil citado en García Canclini, 2004, p. 51).

\section{|La periferia y la desconexión}

El acceso material a la tecnología está intrínsecamente relacionado con el acceso al capital monetario que garantizaría la compra de dispositivos y de acceso a redes ${ }^{11}$. Entonces es fácil concluir que la desigualdad social conlleva a la desconexión y a la "desigual participación en las redes de información (que) se combina con la desigual distribución mediática de los bienes y mensajes de aquellas culturas con las que estamos interactuando" (García Canclini, 2004, p. 190). Sin embargo, lo importante aquí es conocer las particularidades de dicha desconexión que ha sido perpetuada por el destiempo con el que este tipo de comunidades ha transitado los usos y apropiaciones de las TIC analógicas, y actualmente las TIC digitales. Esa desconexión a la sociedad del conocimiento y la información ha conllevado también a la continuidad de las formas de organización comunitaria y política que se han venido retratando, así como a singulares experiencias simbólicas con las TIC: 
Al observar la variedad de compromisos identitarios y de modos de simbolizar el sentido social, comprobamos que los conocimientos necesarios para situarse significativamente en el mundo deben obtenerse tanto en las redes tecnológicas globalizadas como en la transmisión y reelaboración de los patrimonios históricos de cada sociedad (García Canclini, 2004, p. 189).

Es por ello que se propone la importancia de la alfabetización digital como acceso a un conocimiento que ofrece herramientas para el desarrollo de representaciones sociales como productores, difusores y usuarios y la construcción de representaciones mediáticas en los medios sociales. Todo ello en articulación con las particularidades que estas poblaciones han tenido con las TIC en sus lugares de origen rural y posteriormente en la periferia de una ciudad.

En Colombia las grandes extensiones rurales complejizan incluso la conexión territorial y de vías, por lo que ha sido la radio analógica el medio de comunicación por antonomasia y en menor medida los televisores analógicos con señales de televisión abierta. El acercamiento a los celulares inteligentes, los computadores y el acceso al internet suceden principalmente cuando estas comunidades llegan a la ciudad.

Con este panorama es necesario consolidar hábitos de conocimiento que generen el entusiasmo por ser prosumidores y produsuarios; $\mathrm{y}$ en ese accionar fortalezcan la denominada segunda oralidad. Es decir, la posibilidad de registrar en soportes audiovisuales y narrar (haciendo uso de las plataformas digitales) a redes externas sus procesos comunitarios.

En ese sentido García Canclini se pregunta "qué logran estas incorporaciones ocasionales, relativamente aisladas a las redes avanzadas del conocimiento" (García Canclini, 2004, p. 189) a lo que algunos académicos contestarán que no serviría de mucho si se tiene en cuenta que, en plataformas como YouTube, investigaciones demuestran que:

menos del $20 \%$ de los visitantes de YouTube son activos proveedores de contenido, y que 
el $20 \%$ de estos colaboradores cargan el 73\% de los videos (Ding y otros, 2011). En otras palabras, un $4 \%$ de los usuarios de YouTube brinda casi tres cuartos del contenido del sitio, y, por si fuera poco, estos colaboradores activos no son demográficamente representativos en términos de edad y género (Van Dijck, 2016, p. 191).

Además, los algoritmos de estas plataformas potencializan personas (perfiles) y contenidos por encima de otros muchos, direccionando tendencias/viralizaciones $\mathrm{y}$ construyendo influenciadores. Por lo que queda claro que los objetivos de la conexión desde las periferias y por los grupos sociales subalternos que las habitan, no pasa por las dinámicas de las corporaciones propietarias de los medios sociales. Se trata de producir y compartir documentación audiovisual que aporte a la memoria histórica y a la historia social subalterna, la cual difiere en gran medida de la historia social hegemónica construida por los grupos sociales dominantes.

En ese sentido es debatible que "la internet permite establecer una comunicación sin jerarquías, lo que en términos políticos puede traducirse como una comunicación democrática” (Calello Fritzsche, 2007, p. 86). Esto si se piensa los alcances de internet a nivel global o masivo. Además, se estaría obviando que en pleno siglo XXI muchas comunidades siguen desconectadas por la falta de acceso material a los dispositivos y a las redes y carecen de alfabetizaciones propias sobre el funcionamiento corporativo de los medios sociales. Generalmente estos conocimientos de uso y apropiación son empíricos y en destiempo en comparación a aquellos que consolidan las clases sociales medias y altas. Sin embargo, sí es posible "construir espacios de debate alternativos y de articulación de las acciones que llevan a cabo los movimientos sociales" (Calello Fritzsche, 2007, p. 86).

\section{|Metodología}

El umbral teórico de esta investigación propone que las TIC o alguna otra tecnología no tiene vida propia o poderes absolutistas manipulatorios; tampoco que las tecnologías surgen al margen de las necesidades sociales, políticas y económicas de una sociedad: 
Periferias en Cali: alfabetización digital en grupos sociales subalternos en medios sociales digitales Noscué Mera

Las tecnologías son habilitantes (e inhabilitantes), más que determinantes. Aparecen, existen $\mathrm{y}$ desaparecen en un mundo que no es del todo obra suya ... . las tecnologías son cosas sociales, impregnadas de lo simbólico y vulnerables a las eternas paradojas y contradicciones de la vida social, tanto en su creación como en su uso (Silverstone citado en Winocur y Sánchez Vilela, 2016, p. 26).

Si se reconoce que el desarrollo tecnológico está intrínsecamente relacionado con las características de la inserción social que se define por procesos simbólicos en diálogo con contextos puntuales por determinados actores sociales; es necesario definir que el proceso metodológico debe priorizar una

aproximación metodológica que analice los procesos tecnológicos en donde están ocurriendo ... [ello] nos conduce a la construcción de nuevos conceptos y categorías de análisis, que tienden puentes que superan las dicotomías ciegas con que hasta ahora hemos tanteado el tema de las nuevas tecnologías (Santos y Márquez, 2003, p. 79).
Máxime cuando se pretende que los aportes teóricos y metodológicos incluyan las evidencias obtenidas en contextos sociales particulares, históricamente marginados, que generalmente son excluidos de lo que define el conocimiento y la ciencia. Es decir, que lo aportado aquí amplía los conocimientos sobre los usos y apropiaciones de las TIC que difieren ampliamente de las investigaciones basadas en estudios con poblaciones actualizadas, conectadas y pioneras en los accesos a las tecnologías digitales.

Hay antecedentes de investigaciones que concluyen "que no basta comprar y distribuir equipo informativo" (Winocur y Sánchez Vilela, 2016, p. 13) para garantizar la apropiación digital por parte de familias pobres; es necesario asumir un camino intermedio que no desconozca la potencialidad de los sectores populares.

La construcción de representaciones sociales y mediáticas de estos grupos poblacionales en los medios sociales está mediada por los alcances tecnológicos de los aparatos que generalmente son escasos $y$ caracterizada por la desigualdad 
de la alfabetización digital. Pero no se puede obviar el valor simbólico, estético, organizacional y político que aporta la producción audiovisual (fotos, videos, gif, memes, etc.) en los nuevos medios a las luchas sociales y políticas históricas en América Latina. Las clases populares cuentan con influencias coloniales, andinas, afro, campesinas, indígenas; todo ello atravesado por la globalización de modelos neoliberales y capitalistas: "Lejos de ser una hoja en blanco, las familias procesan y filtran las novedades tecnológicas a partir de sus universos simbólicos de pertenencia y experiencia con otras tecnologías" (Winocur y Sánchez Vilela, 2016, p. 26).

En el caso colombiano existió una relación intrínseca entre la epistemología y la escritura de la historia que permite comprender y reconocer "el impacto del racismo en la construcción de alteridades racializadas" entendiendo "que el acceso a la palabra escrita evidenciaba un ejercicio de poder, puesto que el poder nombrar o clasificar la realidad es un modo de poseerlo lingüística $\mathrm{y}$ simbólicamente” (Vega Bendezú, 2013, p. 19). Las elites criollas lograron construir representaciones sociales que connotan los grupos subalternos con adjetivos negativos y de ausencias simbólicas, sociales y políticas.

Por ello la metodología aplicada en estos procesos de investigación debe priorizar el análisis sobre los procesos prácticos y simbólicos que estas comunidades hacen de las TIC, por encima de los planteamientos teóricos que priorizan las posibilidades para acceder a la sociedad de la información como la solución a la inclusión laboral y productiva y/o el mejoramiento a la calidad de vida que permitiría el ascenso social, tal como lo propone el Ministerio TIC en Colombia.

El proceso pedagógico de alfabetización digital como espacio generador de experiencias conjuntas de enseñanza-aprendizaje se caracteriza por el reconocimiento de territorialización híbrido. Exige que cada actividad se articule con las tecnologías de las que se pretende reflexionar y con las características propias de procesos sociales y culturales que caracterizan la comunidad.

Es necesario deconstruir los postulados clásicos de la pedagogía positivista basados en diagnosticar 
el nivel de conocimiento de los sujetos a alfabetizar, para luego clasificarlos y a partir de allí comenzar a transmitir el saber. Por el contrario, se debe tener en cuenta, por un lado, la pedagogía del oprimido propuesta por el pedagogo Paulo Freire, a partir de la cual se reivindica la necesidad de reconocimiento y pertenencia a una determinada clase social como andamiaje de lucha y organización social y política. Se trata de un camino emancipatorio y libertario; es decir, el reconocimiento a la importancia de la construcción de resistencia, autonomía y libertad:

La pedagogía no está limitada a la institución de la educación, si hablamos de pedagogías en general, podemos decir que las luchas que ejercen los movimientos, los colectivos, barrios populares, etc.; muchas veces ejercen un tipo de pedagogía, hacen un tipo de acción-reflexión, la acción para actuar de mejor manera, un continuo proceso de aprendizaje, de desaprendizaje, de reaprendizaje.

pedagogías como metodologías indispensables, necesarias para la transformación social (Walsh en Uniminuto Televisión, 2017).

Por otro lado, la pedagogía decolonial que prioriza el reconocimiento del lugar desde donde se está pensando el conocimiento: América Latina. Se busca la apertura de perspectivas distintas, a partir de procesos continuos en comunidades puntuales que aporten desde sus propias historias y memorias, la construcción del conocimiento. "Lo decolonial no necesita un autor, un manual, recetas o esquemas que digan así es la forma; sino que se trata de ir cuestionando $\mathrm{y}$ preguntando continuamente $\mathrm{y}$ reconocer que existe una estructura de poder" (Walsh en Uniminuto Televisión, 2017).

Con estos antecedentes teóricos, los datos y las experiencias preexistentes construidas con la comunidad, se dio paso a la construcción de los contenidos del proceso pedagógico.

Por ejemplo, fue importante tener en cuenta la problemática que buscaban solucionar en ese momento (febrero-octubre 2017). 
Se trataba de la pavimentación de la carretera de acceso principal a la zona que impedía el ingreso y salida de vehículos en situaciones de urgencia. Teniendo en cuenta que sus prioridades radican en cuestiones tan básicas como el acceso a su vivienda, la obtención de un empleo, la educación básica, la alimentación e incluso la construcción de sus casas; era complejo proponerles la importancia de la construcción de representaciones mediáticas en los medios sociales, entendiéndolos también como espacios de visibilización y lucha. Fue indispensable reflexionar en conjunto con los adultos sobre los usos y apropiaciones que desarrollaron con las Tecnologías de la Información y Comunicación (TIC) precedentes. Con esto se buscaba acercarlos a las nuevas tecnologías, que reflexionaran sobre la posibilidad de fortalecer sus cotidianidades comunicativas digitales. Como se esperaba, fue mucho más fluido el intercambio de experiencias con los jóvenes y los niños, quienes acceden más cómodamente a las dinámicas de la digitalidad.
Se propuso realizar 10 encuentros cada fin de semana, de tres horas cada uno, en un salón comunal en construcción que cobró un valor simbólico y funcional por parte de la comunidad. Los requerimientos técnicos y logísticos que exigió cada sesión fueron gestionados por quien investigaba, pero también por líderes de la comunidad.

Fue revelador que un proceso pedagógico de alfabetización digital no se destacara por la presencia de tecnología, sino que por el contrario emergiera en ese espacio físico la estética rural y las dinámicas propias de mingas o encuentros comunales. Las conversaciones grupales entre mujeres, la importancia de la comida para los niños, la introducción al espacio de tareas como el tejido y la elaboración de artesanías en medio de los ejercicios propuestos.

El desarrollo de los contenidos se dividió en tres bloques, focalizados siempre en ocupar la primera parte de las sesiones a las discusiones teórico-experienciales. Se iniciaba con los aspectos teóricos sobre los medios comunicacionales; para que, durante dicha exposición, 
en conjunto con los asistentes, se dialogara sobre las experiencias que individual, familiar $\mathrm{y}$ comunitariamente habían tenido con las TIC.

Para la segunda parte de las sesiones se proponía la puesta en práctica de la interacción con sus propios dispositivos. La cantidad de asistentes variaba semanalmente entre 25 y 30 personas. Algunos no poseían un dispositivo propio, por lo que era necesario consolidar equipos de trabajo.

En el primer bloque se estudió la historicidad y el desarrollo tecnológico de las TIC. Pasando por la oralidad y la escritura, la prensa, la radio y la televisión analógicas y finalmente la Internet; donde se concluyó que convergen todas las anteriores y las nuevas que se catalogan como medios sociales. Se propuso comprender que las TIC no tienen fecha de vencimiento, ni paredes divisorias entre unas y otras. Se reconoció que el destiempo con e l que estas poblaciones han llegado a la interacción y posterior utilización de las TIC digitales, es abismal. Por ejemplo, aunque Facebook y YouTube funcionan desde el año 2004 y 2005; fue solo hasta hace poco más de un par de años que estas poblaciones conocieron sobre su existencia. Por su parte, la televisión por demanda como Netflix solo está dentro de su universo simbólico, efímeramente. Por eso en una de las sesiones visualizaron el capítulo "Caída en Picada" de la serie televisiva Black Mirror (Jones, Schur, Brooker y Wright, 2016). Las críticas al comportamiento desmedido de la protagonista en sus redes sociales digitales no se hicieron esperar.

Durante el inicio de este primer bloque se revisaron las representaciones mediáticas de la comunidad existentes en los medios sociales, puntualmente en YouTube. Se encontraron diversos videos de noticias en el canal de YouTube del noticiero local independiente Pazífico Noticias (2014, 2015, 2016) donde se informa sobre la problemática del reconocimiento legal de los terrenos que habita la comunidad. También se visualizó una nota que el mismo noticiero hizo, meses antes del proceso de alfabetización digital. Este video (Pazífico Noticias, 2017) fue el resultado de una actividad que se propuso en el marco de esta 
investigación. Se tramitó ante la producción del noticiero la visita de sus reporteros para que evidenciaran la problemática de la pavimentación de la carretera principal que estaba siendo gestionada por la misma comunidad, exponiendo entonces ante la sociedad el abandono estatal, pero también las formas de organización comunitaria que ocurren en las periferias de la ciudad.

También se encontró un video en el canal de la Fundación Internacional Save The Children (2013) donde narran el apoyo que la fundación hace en la zona con purificadores de agua y programas de alfabetización sobre nutrición infantil. Cabe aclarar que la constante estética y el discurso que predomina en los primeros videos de Pazífico Noticias y el de la Fundación Save the Children está relacionada con las carencias y las ausencias en las que vive la comunidad: calles embarradas, casas de bareque, ausencia de legitimidad en los terrenos que habitan, etc. Sin embargo, la nota que se logró en el marco de la investigación tuvo en enfoque discursivo diferente. Más allá de denunciar el abandono estatal, se priorizó evidenciar la organización y la autogestión que esta comunidad hace en pos de su bienestar. Por supuesto que la estética visual seguía exponiendo las calles embarradas que faltan por pavimentar, pero también mostraba la calle principal que ya estaba pavimentada.

También es necesario apuntar que en el desarrollo de las pesquisas anteriores al proceso pedagógico, se buscó proyectos comunicativos preexistentes. En esa pesquisa se encontró el video producido por la usuaria María Eugenia titulado "video cabildo indígena nasa Buenavista” (2013), que tiene una duración de 8:54 minutos y cuenta con 1 mil 718 vistas. En el pie del video se puede leer:

LO ANCESTRAL EN EL CABILDO INDÍGENA NASA BUENAVISTA

Danza y música

Tejido

Proyecto Productivo Tejiendo Comunicación.

Actualmente en el cabildo indígena Nasa Buenavista la mayoría de la población se comunica en su lengua nativa, Nasa Yuwe; mantiene 
Periferias en Cali: alfabetización digital en grupos sociales subalternos en medios sociales digitales

vivas tradiciones como medicina tradicional, ritualidades, minga, trueque, así como diferentes expresiones artísticas autóctonas como música, danza y tejidos artesanales.

$\mathrm{Su}$ interés es fortalecerse como comunidad y mantener vivas las tradiciones de sus ancestros, por lo que están en la búsqueda de acceder e implementar procesos de educación propia que contribuyan en ese aspecto y medien los intercambios entre Nasas y demás comunidades.

Habiendo conocido sobre los antecedentes de las representaciones mediáticas preexistentes en los medios sociales; se continuó priorizando sobre la importancia del reconocimiento socio-cultural como base para comenzar a construir un perfil identitario digital: la reivindicación del lugar de origen, de las costumbres tradicionales $y$ de las experiencias vividas en el nuevo territorio urbano; todo ello en articulación con las representaciones mediáticas que estarán en la web gracias al uso posible de las redes sociales digitales, como proceso constructor de la identidad individual, pero también como aporte a la construcción de representaciones comunitarias. Los productos audiovisuales de este primer bloque fueron videos selfie y notas periodísticas sobre las mingas en el barrio. Los asistentes los presentaron, grabaron y subieron a la red.

Para la realización de las notas periodísticas (Collazos, 2017a, 2017b) se dividieron dos grupos de trabajo y al interior de cada grupo se repartieron las funciones necesarias. Las reporteras que presentaban la nota y quienes hacían la cámara con sus teléfonos celulares. La estética visual priorizó mostrar las formas de trabajo comunitario que estaban ocurriendo ese domingo en la zona. Se construyó la información, de mano de las y los colaboradores de las mingas, para conocer de primera mano los objetivos de los trabajos que se adelantaban. Se trataba de una minga que se ocupaba de rellenar un espacio de la zona para posteriormente hacer una cancha de futbol para los niños y en la otra minga se ocupaban de ampliar la carretera a una zona específica para que puedan pasar los vehículos que 
traen materiales para la construcción de las casas.

El segundo bloque del proceso pedagógico se ocupó de ampliar los conocimientos frente a los planteamientos básicos sobre la composición de la imagen en foto y en video. Se reconoció que los contenidos en los medios sociales no se caracterizan, necesariamente, por la alta calidad de imagen; sino por los valores simbólicos $\mathrm{y}$ funcionales del mensaje. Los ejercicios variaron entre conocer y experimentar la producción de imágenes teniendo en cuenta los planos cinematográficos, el manejo de la luz e incluso se dedicó una sesión técnica sobre la sensibilidad sonora necesaria para el registro de video; todo ello priorizando siempre la estética propia del barrio y sus personajes: los árboles plantados al frente de las casas, gallinas caminando por los andenes, las puertas de las casas abiertas, las radios sintonizadas en emisoras Caucanas que programan músicas campesinas y populares.

El último bloque estuvo dedicado a conocer el funcionamiento de la Red Social (Facebook) y la Plataforma
Digital (YouTube) más relevantes (Van Dijck, 2016); así como también se dedicó tiempo para dialogar sobre algunas Aplicaciones (Giphy) $\mathrm{y}$ otras herramientas que facilitan la producción de memes y gif. Estos productos visuales exigen propuestas escénicas, por lo que una de las sesiones estuvo dedicada a la expresión corporal; generando así un nivel de confianza individual y grupal que aportó a la producción creativa. De esa forma se produjeron gif que evidencian sus conocimientos laborales con el tejido, la costura y las artesanías (Alto Nápoles en Red, 2017a, 2017b, 2017c). Por su parte, los niños y niñas propusieron expresiones corporales con bailes y juegos (Alto Nápoles en Red, 2017d). También se motivó la producción de videos tutoriales y se aprendió a crear el canal en YouTube para poder compartirlos, además de descargar videos que pudiesen ser de su interés. Se conversó sobre los nuevos símbolos (emojis, emoticones), signos $\mathrm{y}$ lenguajes en los medios sociales.

Finalmente se propuso un ejercicio que reuniera los conocimientos experimentados en el proceso pedagógico y sus historias de 
vida. Cada participante narró ante la cámara una historia que pudiese ser producida como ficción en un filminuto que se realizaría en equipo. Todas las historias estuvieron relacionadas con anécdotas o situaciones vividas en sus lugares de origen o cuando llegaron a su nuevo territorio, relacionaron su pasado con su presente. Estas producciones se encuentran consignadas en un cortometraje documental (Alto Nápoles en Red, 2018) que expone audiovisualmente este proceso de alfabetización digital.

\section{|Conclusión}

Las desigualdades históricas que estos grupos sociales han transitado no se relacionan fundamentalmente con usar $y$ aplicar las tecnologías como medios en sus vidas para ascender de clase social. El destiempo que vivieron quienes hoy son adultos con la televisión por suscripción es lo que quienes son jóvenes hoy viven con el desconocimiento 0 el no disfrute de la televisión por demanda y el consumo de series; o lo que quienes son niños hoy viven al no tener dispositivos actualizados, conexiones estables y constantes o simplemente multipantallas que amplíen su experiencia con los consumos culturales mediáticos.

El proyecto pedagógico de alfabetización digital como metodología en las investigaciones sociales y comunicacionales permite proponer que las políticas públicas de las TIC tendrán que incorporar la importancia del territorio como espacio constructor de representaciones digitales.

La posibilidad de que los grupos sociales subalternos produzcan representaciones mediáticas en los medios sociales, a partir de los usos y apropiaciones de las TIC en el marco de experiencias comunitarias, aporta al fortalecimiento de las redes comunitarias y a la construcción de un activismo digital que conlleve a la consolidación de representaciones sociales como productores, difusores y usuarios en las redes sociales y las plataformas digitales.

Winocur y Sánchez Vilela (2016) compartían que las madres de los niños que recibieron la 
computadora Ceibal en Uruguay sentían temor de tocar y mucho menos manipular la máquina por miedo a arruinarla. En esta investigación se pudo constatar que si se genera un espacio pedagógico que acerque las posibilidades de usos y apropiaciones que pueden desarrollar a partir de la interacción, los adultos que saltaron de la televisión analógica a los medios sociales, sin pasar por el computador, tendrán confianza de experimentar online porque se requiere habilidades básicas, pero no simples como saber leer y escribir para lograr producir y publicar.

La experiencia desarrollada permite vislumbrar que es posible la generación de autorepresentaciones mediáticas en los medios sociales de parte de grupos subalternos que aporten a la memoria histórica como discurso de luchas y resistencias a la construcción de representaciones sociales propuestas desde el discurso hegemónico. Quizá no para alcanzar la popularidad online o la influencia global; pero sí para aportar a la construcción de la historia social subalterna.

\section{|Notas al final}

1 Es de conocimiento empírico que muchas personas que atravesaron este flagelo, no hicieron el reconocimiento ante el Estado por diversas razones como el temor a represalias por parte de los grupos ilegales. Entonces se infiere que existen muchas más personas en esta condición.

2 Se considera el uso de esta denominación conceptual a partir de los postulados teóricos que realizó el intelectual italiano Antonio Gramsci, en torno al reconocimiento de diversos grupos sociales insertos en estructuras de poder político, económico y sociocultural que devienen en diversas luchas simbólicas que definen la subalternidad y la hegemonía de dichos grupos sociales (Green, 2004; Modonesi, 2010).

3 Se reconoce la representación como concepto interpretado teóricamente desde acepciones con tradiciones lingüísticas, discursivas, socio-políticas; para luego complementarlo desde la interpretación del concepto de cultura como resultado de la construcción de prácticas de significación (fuertemente sedimentadas) presentes en todo proceso social que es posible por lo territorial, lo político y lo económico (Grimson, 2011) además de lo tecnológico, tal como se plantea en esta investigación.

4 Se reconoce el concepto de representación mediática como "representaciones producidas por los medios de comunicación, entendiéndolos como operadores de visibilidad y traducibilidad, y responsables junto con otros sistemas simbólicos de trazar mapas, organizar y engarzar el ordenamiento social histórico en la dimensión de la cultura" (Cebrelli y Rodríguez, 2013, p. 90). 
5 Se explicita que el reconocimiento que se hace de este concepto en esta investigación está intrínsecamente relacionado con el concepto de cultura participativa propuesto por Jenkins (1992) y a partir del cual plantea una oposición entre la industria de los medios y la actividad colaborativa de individuos insertos en grupos o redes amplias, desde donde les es posible propagar contenido más allá de su proximidad geográfica. Es decir, el reconocimiento de valor simbólico y de disputa representacional a partir de la producción de bienes culturales en los espacios digitales.

6 Se reconoce la alfabetización digital como un proceso pedagógico direccionado a la comprensión y posterior utilización del lenguaje multimedial, es decir, un proceso que conlleve a "la capacidad de comprender y utilizar la información de fuentes diversas y múltiples formatos, cuando se presenta a través del ordenador" (Gutiérrez Martín, 2003, p. 4) y los dispositivos móviles. Así mismo, se resalta la necesidad de rescatar y vincular las particularidades históricas socioculturales, educacionales y de acceso a las TIC que han tenido los grupos sociales con los que se desarrolle el proceso pedagógico. Esta recomendación pedagógica direccionada a la emancipación y organización social se reivindica desde los postulados de la educación popular y decolonial propuesta por autores como Paulo Freire (1990) y Catherine Walsh (2003).

7 Minka es propio del idioma del pueblo indígena Yanacona del Cauca; al cual pertenecen muchos habitantes de Brisas de Alto Nápoles.

8 Las JAC son organizaciones barriales, reconocidas legalmente para recibir y distribuir recursos públicos.

9 El Cabildo Indígena Nasa Alto Buenavista no es reconocido por el gobierno municipal como cabildo urbano, porque argumentan que ya hay reconocimiento de un cabildo urbano Nasa que debe acoger a todos los Nasas residentes en Cali.

10 La comunidad Yanacona no busca ser reconocida como cabildo. Se adhieren al Cabildo Urbano Yanacona de Cali.

11 Colombia no cuenta con una política pública, como lo fue el "Plan Ceibal" en Uruguay o "Plan Conectar Igualdad" en Argentina. 


\section{|Referencias}

Alto Nápoles en Red. (Productor). (2017a). Doña Mimia la tejedora. [Archivo GIF]. Recuperado de https://giphy.com/gifs/latejedora-altonpolesenreddoamimia-3ov9jQJEKTJaUo9geA

Alto Nápoles en Red. (Productor). (2017b). Mariela la costurera. [Archivo GIF]. Recuperado de https://giphy.com/gifs/alto-napoles-en-red-mariela3ohhwEGzVSEczi9E3K

Alto Nápoles en Red. (Productor). (2017c). Yuli nuestra artesana en Alto Nápoles. [Archivo GIF]. Recuperado de https://giphy.com/gifs/yuliartesana-alto-npoles-en-red-3ohhwKAGXzeg0ItKH6

Alto Nápoles en Red. (Productor). (2017d). Gifs en Alto Nápoles en Red. [Archivo GIF]. Recuperado de https://giphy.com/gifs/gifs-en-alto-napolesred-11J9JNIZHtWYqMzAs

Alto Nápoles en Red (Productor). (2018). ALTO NÁPOLES EN RED: Proyecto Pedagógico de Alfabetización Digital. [Video de YouTube]. Recuperado de https://www.youtube.com/watch?v=sb3WEAeDyBU

Calello, T. \& Fritzsche, F. (2007). Internet como mediadora en la construcción de redes de información y comunicación de las asambleas vecinales. En A. Quintar, T y Calello, G. (Eds.), Los usos de las TICs: Una mirada multidimensional (pp. 85-112). Buenos Aires, Argentina: Prometeo Libros y Universidad Nacional General Sarmiento.

Cebrelli, A. \& Rodríguez, M. G. (2013). ¿Puede (in)visibilizarse al subalterno? Algunas reflexiones sobre las representaciones y los medios. Trampas de la Comunicación y la Cultura, 76-98.

Collazos, H. (Productor). (2017a). Minga comunitaria en Alto Nápoles Bosques 2. [Video de YouTube]. Recuperado de https://www.youtube.com/ watch?v=yuYjajEyKYE 
Periferias en Cali: alfabetización digital en grupos sociales subalternos en medios sociales digitales Noscué Mera

Collazos, H. (Productor). (2017b). Minga domingo 24 de sept en Alto Nápoles. [Video de YouTube]. Recuperado de https://www.youtube.com/ watch?v=oWT8TZRyX8I

Colombia sigue siendo el país con más desplazados internos: 7,4 millones. (18 de junio de 2017). El Espectador. Recuperado de https://www. elespectador.com/noticias/el-mundo/colombia-sigue-siendo-el-pais-conmas-desplazados-internos-74-millones-articulo-698945

De cuatro millones de desplazado en Colombia, 80 mil viven en Cali. (20 de junio de 2012). El Pueblo. Recuperado de http://elpueblo.com.co/de-cuatromillones-de-desplazados-en-colombia-80-mil-vivenn-en-cali/

Freire, P. (1990). La Pedagogía del Oprimido. Buenos Aires, Argentina: Siglo XXI.

García Canclini, N. (2004). Diferentes, desiguales y desconectados: Mapas de la interculturalidad. Barcelona, España: Gedisa S.A.

Green, M. E. (2004). Método y análisis de Gramsci sobre los grupos subalternos. En D. Kanoussi (Ed.), Poder y hegemonía hoy: Gramsci en la era global (pp. 63-78). México, D.F, México: Plaza y Valdés, S.A. de C.V.

Grimson, A. (2011). Los límites de la cultura: Crítica de las teorías de la identidad. Buenos Aires, Argentina: Siglo XXI Editores.

Gutiérrez Martín, A. (2003). Alfabetización digital: algo más que ratones y teclas. Bogotá, Colombia: Gedisa.

Jenkins, H. (1992). Textual Poachers: Television Fans and Participatory Culture. Studies in culture and communication. New York, NY: Routledge.

Jones, R., Schur M., Brooker, C. (Escritores), \& Wright, J. (Director). (2016). Caída en Picada [Episodio de serie de televisión]. En C. Brooker (Productor), Black Mirror. Los Gatos, C.A.: Netflix.

Los departamentos de Colombia con mayor número de desplazados. (20 de junio de 2016). El Espectador. Recuperado de https://www.elespectador. com/noticias/nacional/los-departamentos-de-colombia-mayor-numerode-deplazado-articulo-638967 
María Eugenia. (Productora). (2013). Video cabildo indígena nasa Buenavista. [Video de YouTube]. Recuperado de: https://www.youtube.com/ watch?v=YGSWcAcUOWI

Modonesi, M. (2010). Subalternidad, antagonismo y autonomía: Marxismos y subjetivación política. Buenos Aires, Argentina: CLACSO.

Pazífico Noticias. (Productor). (2014). Indígenas Nasa y Yanaconas reclaman tierras en el sur de Cali. Recuperado de https://www.youtube.com/ watch?v=arcSh3KY6J8

Pazífico Noticias. (Productor). (2015). Programas de inversión social reclaman en las laderas de Cali. [Video de YouTube]. Recuperado de https://www. youtube.com/watch?v=A-qSGwaNBc8

Pazífico Noticias. (Productor). (2016) En Alto Nápoles, 68 familias indígenas se rebelan contra el abandono del Municipio. [Video de YouTube]. Recuperado de https://www.youtube.com/watch?v=BgY39wGSO0A

Pazífico Noticias (Productor). (2017). Vía de Alto Nápoles se abre paso gracias a la comunidad; la población reclama ayuda de la Alcaldía. [Video de YouTube]. Recuperado de https://www.youtube.com/watch?v=zU14nbFImEI\&t=1s

Quintar, A. (2007). Redes sociales y comunidades virtuales. En A. Quintar, T. Calello y G. Aprea (Eds.). Los usos de las TICs: una mirada multidimensional (pp. 71-84). Buenos Aires, Argentina: Prometeo Libros y Universidad Nacional General Sarmiento.

Santos, M. J. \& Márquez, M. T. (2003). Trayectorias y estilos tecnológicos: Propuestas para una Antropología de la tecnología. En C. Bueno y M. J. Santos (Eds.). Nuevas tecnologías y cultura (pp. 75-97). México D.F., México: Anthropos.

Save The Children Colombia. (Productor). (2013). Historia de vida Alto Nápoles. [Video de YouTube]. Recuperado de https://www.youtube.com/ watch?v=HbNWvTtjepM\&t=161s 
Uniminuto Televisión. (Productor). (2017). Oiga profe/Pedagogías Decoloniales/ Catherine Walsh. [Video de Youtube]. Recuperado de https://youtu.be/ j6FNfOdh7tU

Van Dijck, J. (2016). La cultura de la conectividad: Una historia crítica de las redes sociales. Buenos Aires, Argentina: Siglo Veintiuno Editores.

Vega Bendezú, M. (2013). Discursos sobre “raza” y nación en Colombia, 18801930. Santiago de Cali, Colombia: Unidad de Artes Gráficas de la Facultad de Humanidades, Universidad del Valle.

Vio, M. \& Fritzsche, F. (2007). Las nuevas Tecnologías de la Información y Comunicación en el territorio: en el umbral de una ciudad sin fin. En A. Quintar, T. Calello y G. Aprea (Eds.). Los usos de las TICs: Una mirada multidimensional (pp. 39-70). Buenos Aires, Argentina: Prometeo Libros y Universidad Nacional General Sarmiento.

Walsh, C. (2003). Estudios culturales latinoamericanos retos desde y sobre la región andina. En C. Walsh (Ed.) Estudios Culturales Latinoamericanos. Retos desde y sobre la región andina. Quito, Ecuador: Universidad Andina Simón Bolívar Abya-Yala.

Winocur, R. \& Sánchez Vilela, R. (2016). Familias pobres y computadoras: Claroscuros de la apropiación digital. Montevideo, Uruguay: Editorial Planeta S.A. 